\title{
STATISTICAL EVALUATION OF LARGE-SCALE DATA LOGISTICS SYSTEM
}

\author{
Radovan Šomplák, Zlata Šmídová, Veronika Smejkalová, Vlastimír Nevrlý \\ Brno University of Technology, Faculty of Mechanical Engineering \\ Institute of Process Engineering \\ Technická 2896/2, 61669 Brno \\ Czech Republic \\ Radovan.Somplak@vutbr.cz
}

\begin{abstract}
Data recording is struggling with the occurrence of errors, which worsen the accuracy of follow-up calculations. Achievement of satisfactory results requires the data processing to eliminate the influence of errors. This paper applies a data reconciliation technique for mining of data from recording movement vehicles. The database collects information about the start and end point of the route (GPS coordinates) and total duration. The presented methodology smooths available data and allows to obtain an estimation of transportation time through individual parts of the entire recorded route. This process allows obtaining valuable information which can be used for further transportation planning. First, the proposed mathematical model is tested on simplified example. The real data application requires necessary preprocessing within which anticipated routes are designed. Thus, the database is supplemented with information on the probable speed of the vehicle. The mathematical model is based on weighted least squares data reconciliation which is organized iteratively. Due to the timeconsuming calculation, the linearised model is computed to initialize the values for a complex model. The attention is also paid to the weight setting. The weighing system is designed to reflect the quality of specific data and the dependence on the frequency of traffic. In this respect, the model is not strict, which leaves the possibility to adapt to the current data. The case study focuses on the GPS data of shipping vehicles in the particular city in the Czech Republic with several types of roads.
\end{abstract}

Keywords: data reconciliation, GPS data, random errors, weight of data, transportation time

\section{Introduction}

The logistic issues are solved in various areas of human activities and in many branches, it covers a significant part of the costs. The current logistics system is very often ineffective. However, the technology development allows improving the system performance. The smart chips can be located at the service points, as it is presented in [1], where the sensor-based networks monitor the waste load in a container to improve the collection efficiency, or directly in the vehicle. Currently, many companies collect data about vehicle movements in real time (commonly the GPS is used). Such databases provide a potential for improvement of transport infrastructure and for reducing transport costs. Even though modern technology brings a high-efficiency potential, these services are not always available. The article [2] describes methods to detect electronically the driver's intention to change the route for consequential optimization, eventually to detect vehicle position in localities with weak or no GPS signal. The compromises between velocity data collected from GPS in probe vehicles and velocity data obtained from inductive loop detectors were sought in [3]. The obtained measurements were combined with a mathematical traffic model using a data assimilation technique. Based on their case study, the dynamic travel time estimation can be achieved with less than $10 \%$ error. The amount of recorded information is in relation with the costs of recording. The paper [4] investigates the question, how much GPS data is needed to provide accurate speed information. The findings also indicate that data fusion makes possible the effective use of data from multiple sources. In general, the amount of data needed depends on the quality of the data in terms of the sample rate.

The occurrence of errors is very common for data recording. The technique called data reconciliation deals with data adjusting and accuracy improving, see [5]. This technique automatically edits data with complying certain constraints. Errors can be divided according to their origin on systematic, gross and random. The systematic errors are more predictable. These errors tend to have a constant value with repeated measurements under the same conditions. The gross errors, so-called outliers, usually arise from human or equipment failure. The paper [6] eliminates these type of inaccuracies in waste production data by minimizing the absolute value of errors. It represents a robust approach which fits on the dataset. The gross error in data reconciliation was of interest in [7]. The presented method combines two principles. The principal component analysis detects the 
gross errors, which is followed by the use of an appropriate function. Another approach is proposed in paper [9], it provides the framework for gross error detection in power plants through data reconciliation.

Sometimes, when a measurement of a process is repeated under identical conditions, the same value is not obtained. This property is studied in the paper [8], which deals with multi-axis machine error modelling. Since the monitoring is not absolutely accurate and the random errors are observed, datasets are usually corrected through weighted least squares method. Assuming normal distribution with zero mean for measurement errors, the software STAN can be used to implement algorithm performing nonlinear data reconciliation [10]. This procedure works with the known covariance matrix of random errors. The two-step strategy for gross error detection and data reconciliation was described in [11]. First, the gross errors are detected using their prior probabilities and second, the data reconciliation is performed. The paper [12] compares the two common methods of data reconciliation - Kalman filter and quadratic program employed in the pipeline network. On the other hand, in [13], the data of natural gas processing were reconciled to satisfy mass balance equations. An important problem related to realistic decision-making is incomplete data sets. The paper [14] coped with this problem by presenting a heuristics algorithm to generate missing input information using decision tree topologies and integrates it into incomplete data sets.

Most of the above-mentioned papers consider the covariance matrix as known prior to the calculation. When the matrix is unavailable, it is necessary to appropriately design the relation in the matrix. This paper deals with the transportation task aimed at estimating the average speed for individual infrastructure segments. It presents the reconciliation with unknown covariance matrix applied on GPS data from a particular city. The probable complete route is compiled including transit points according to information about starting and ending points of routes. The entire routes are separated into so-called segments in the strategical places (usually crossroads). The principle is illustrated in the motivation Fig. 1. The mathematical model for the data reconciliation is presented in the Sect. 3. The application of the model with real data requires the consideration of other data properties and time requirements are necessary. The heuristic methodology is suggested to solve the mentioned task as it consists of four phases:

- preprocessing: identifying the probable path, road type detection and maximal allowed speed, outliers removal,

- initialization: solving the linearised model to set initial estimates,

- weight setting,

- quadratic model application.

The results provide an estimation of probable time spent on each segment and it can give an detail information about transportation in a particular area. The results can be used for further calculation, i.e. in the paper [15], which suggested the approach for facility location problem, where the waste collection cost is minimized. This opens up opportunities for further improving the transport infrastructure.

\section{Task Definition}

Vehicle movement recording is being used in the context of the transportation organization in any city. The available data source of this paper provides information about the start and end point of each route, the time spends on this route, average speed and maximal speed. The presented approach suggests dividing each route into several segments, which can be defined by crossroad. The aim of this contribution is to utilize existing dataset to assess the duration of different routes, which are formed by segments. The transit time of each segment should be based on data from routes. This time estimation of new routes could be a valuable information for logistics planning in various sectors. A very simplified situation is illustrated in the scheme Fig. 1.

The Fig. 1 shows the situation with six routes, which are differentiated by colours and each route consists of several segments notated by 1 st segment - 5th segment. These segments are defined by crossroads. In the real application, only information about the total time of routes is available. Outputs of the methodology should give estimated segment transition time. To verify the suitability of the model, durations for all routes are stated in the simplified example. The aim is to gain these values within the computation. The time, which each route spent on the corresponding segment, is given by numbers in the Table 1 (the colour of number corresponds with the colour of the route). The time spent on each segment varies for different routes, because real transportation time also fluctuates depending on the circumstances of the traffic. The input information is summarized in the Table 1.

The aim is to suggest the model, which calculates the approximate time spent on each segment (1st - 5th segment). The estimate should be close to the average passing time (regarding all the respective routes) and also between the minimum and maximum transit time of each segment, which can be given by speed limits. This back check could provide an information whether the approach works properly. 


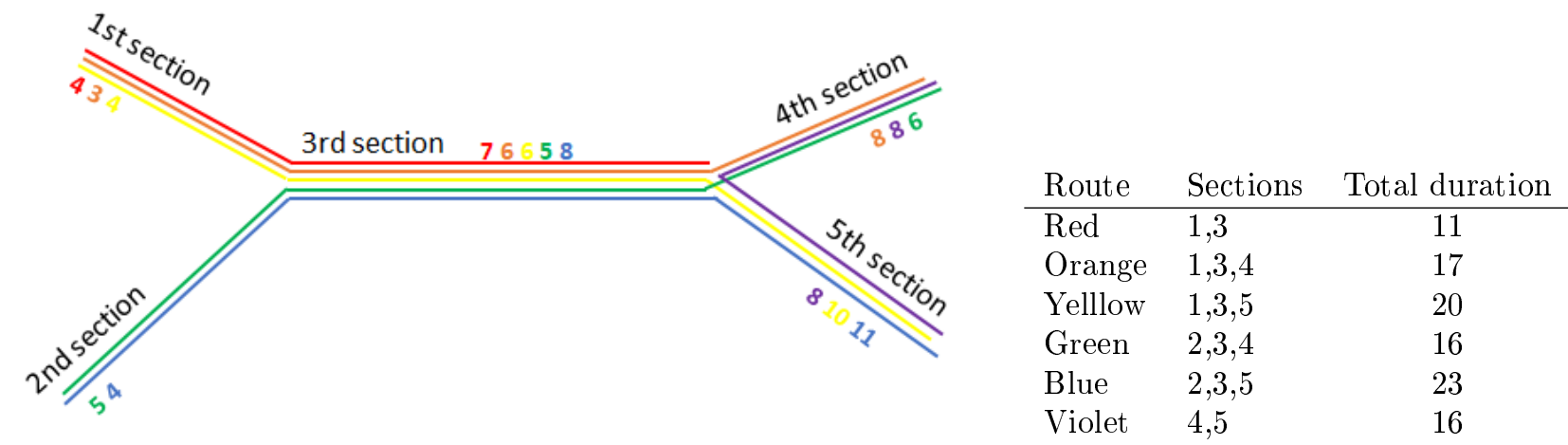

Figure $1 \&$ Table 1: Schematic representation of routes and segments on the simplified example

\section{Mathematical Model}

The mathematical model is built in order to estimate the time required for transit through individual segments of the transportation network. The available database gives information about total time of the whole route, the principle is based on data reconciliation least squares method.

\subsection{Notation}

\section{Sets}

$i \in I \quad$ routes (consisting of segments), $\quad j \in J \quad$ segments of the routes.

\section{Parameters}

$T_{i} \quad$ total time of transit through the route $i, \bar{t}_{j}$ $t_{j}^{\max }$ maximum time spent on a segment $j, \quad t_{j}^{\text {min }}$ average time spent on a segment $j$, $v_{j} \quad$ weight reflecting variability of data, $t_{j}^{\text {min }}$ minimum time spent on a segment $j$,

$A_{i, j} \quad$ matrix indicating relationships between route $i$ and segment $j$.

\section{Decisions variables}

$t_{i, j} \quad$ time spent on the segment $i$ of route $j$,

$p_{i, j}^{\max ,+}$ positive penalty for exceeding maximum time,

$t_{j}^{o} \quad$ optimal time spent on the segment $j$,

$p_{i, j}^{m i n,+}$ positive penalty for exceeding minimum time, $\quad p_{i, j}^{m i n,-}$ negative penalty for maximum time, $\delta_{i, j}$ time deviation for route $i$ and segment $j$. negative penalty for minimum time,

\subsection{Model Formulation}

$$
\min \sum_{i \in I} \sum_{j \in J} \delta_{i, j}^{2}
$$

s.t.

$$
\begin{aligned}
T_{i} & =\sum_{j \in J} A_{i, j} t_{i, j}, \\
t_{j}^{o} & =t_{i, j}+\delta_{i, j}, \\
t_{i, j} & \geq 0 \\
t_{j}^{o} & \geq 0
\end{aligned}
$$

$$
\begin{array}{r}
\forall i \in I, \\
\forall i \in I, \forall j \in J, \\
\forall i \in I, \forall j \in J, \\
\forall j \in J .
\end{array}
$$

The objective function (1) ensures the balanced estimation of transit time $t_{j}^{o}$ through the segments $j$ using minimization of squared deviations $\delta_{i, j}$. The input data of route duration is distributed among corresponding segments and their time is corrected in order to assess final transit time. The constraint (2) connects the total time on the route $T_{i}$ and duration of its individual segments $t_{i, j}$ and matrix $A_{i, j}$ assigns segment $j$ with route $i$. The estimation of transit time $t_{j}^{o}$ for segment $j$ is given by specific duration for route $t_{i, j}$ and corresponding deviation $\delta_{i, j}$. The time values both $t_{i, j}$ and $t_{j}^{o}$ are stated as non-negative (4), (5). 


\subsection{Testing of the Model on a Simplified Example}

The model presented in the Sect. 3 was applied to the input data from the Table 1 and the results are summarized in the Table 2. It can be observed, that the mathematical model in the presented form is not able to estimate the time required for individual segments and the value $t_{j}^{o}$ is significantly different from the average $\bar{t}_{j}$. The average is known only for the simplified example for the verification reason. The difference between average and final transit time is caused by minimizing overall error given by elements $\delta_{i, j}$. Therefore the very short time is assigned to some segments, specifically to the first and second segment.

Table 2: The summarization of results of simlified example [min]

\begin{tabular}{llccccc} 
& & \multicolumn{5}{c}{ Segment } \\
& Route & 1st & 2nd & 3rd & 4th & 5 th \\
\hline Errors & Red & -0.125 & - & -0.125 & - & - \\
& Orange & -0.271 & - & -0.271 & -0.271 & - \\
& Yellow & 0.396 & - & 0.396 & - & 0.396 \\
& Green & - & 0.333 & 0.333 & .333 & - \\
& Blue & - & -0.333 & -0.333 & - & -0.333 \\
& Violet & - & - & - & -0.063 & -0.063 \\
\hline$t_{j}^{o}$ & & 0.396 & 1.208 & 10.354 & 5.437 & 10.438 \\
$\bar{t}_{j}$ & & 3.667 & 4.500 & 6.400 & 7.333 & 9.667
\end{tabular}

In order to maintain realistic outputs, additional constraints were suggested for the model. The minimal and maximal time spent on each segment is limited with respect to input data of illustrative example in the Table 1. Exceeding the specified bounds results in a penalty, which is integrated into the objective function. Suggested constraints are given by (6) - (8).

$$
\begin{aligned}
p_{i, j}^{\max ,+}-p_{i, j}^{\max ,-} & =t_{j}^{\max }-t_{i, j}, & & \forall i \in I, \forall j \in J, \\
p_{i, j}^{\min ,+}-p_{i, j}^{\min ,-} & =t_{i, j}-t_{j}^{\min }, & & \forall i \in I, \forall j \in J, \\
p_{i, j}^{\max ,+}, p_{i, j}^{\max ,-}, p_{i, j}^{\min ,+}, p_{i, j}^{\min ,-} & \geq 0, & & \forall i \in I, \forall j \in J .
\end{aligned}
$$

The (6) indicates the penalty for exceeding the maximal time and the (7) applies the same for not achieving the minimum time. Each variable connected with penalty have to be non-negative (8). The limitations of time spent on individual segments are summarized in the Table 3 for the simplified example from Fig. 1. Bounds might be set according to speed limits or traffic experience.

Table 3: Limitation of minimal and maximal time on segments [min]

\begin{tabular}{ccc} 
segment & $t^{\min }$ & $t^{\max }$ \\
\hline 1st & 3 & 6 \\
2nd & 4 & 5 \\
3rd & 5 & 9 \\
4th & 6 & 9 \\
5th & 8 & 12
\end{tabular}

The estimation have to lie within these bounds, otherwise the exceeding is penalised in the objective function. Therefore, the objective function is transformed into the following form:

$$
z=\sum_{i \in I} \sum_{j \in J}\left(\frac{w_{j}^{2}}{v_{j}} \delta_{i, j}^{2}+\left(p_{i, j}^{\max ,-}\right)^{2}+\left(p_{i, j}^{\min ,-}\right)^{2}\right),
$$

where $w_{j}$ and $v_{j}$ are weights. Within the model, the option of different requirement on the dependency of results on available data amount is retained. The square of deviation $\delta_{i, j}$ is weighted by $w_{j}$. The weight $w_{j}$ can be adapted according to the links between errors and traffic data frequency as in the following two equations:

$$
\begin{array}{ll}
w_{j}=\frac{\sum_{i \in I} A_{i, j}}{\max _{j}\left(\sum_{i \in I} A_{i, j}\right)}, & \forall j \in J, \\
w_{j}=\frac{1}{\sum_{i \in I} A_{i, j}}, & \forall j \in J .
\end{array}
$$


In the case of traffic frequency dependence, the weight in the form (10) is used. With this weight setting, the model prefers segments with a higher amount of routes passing them. The independence on traffic frequency is ensured by (11), where the weight $w_{j}$ normalizes the input data. It depends on the particular application, which approach is chosen. The data quality should be also taken into account with regards to weight setting. Data with higher variability are considered as less responsible and the inverted value of variance appears in the objective function (1). Regarding the real data, the parameter $\bar{t}_{j}$ is unknown prior to the calculation and so the problem is solved iteratively.

$$
v_{j}=\frac{\sum_{i \in I}\left(\bar{t}_{j}-t_{i, j}\right)^{2}}{|I|}, \quad \forall j \in J .
$$

\subsection{Results of a Simplified Example}

The example introduced in the previous parts is used to verify the functionality of the model in this section, because the travel duration on each segment is actually known, as in Table 1. The results of simplified example are summarized in the Table 4, which shows the errors on each segment. It can be observed, that the optimal value $t_{j}^{o}$ is strikingly close to average values on each segment $\bar{t}_{j}$. The results of the test example have reached the expected values and the model in this form satisfies the requirements of the task. In the following section, it is applied to real waste transportation data.

Table 4: The summary of results of weighted model [min]

\begin{tabular}{llccccc} 
& & \multicolumn{5}{c}{ Segment } \\
& Route & 1st & 2nd & 3rd & 4th & 5 th \\
\hline Errors & Red & -0.045 & - & -0.189 & - & - \\
& Orange & -0.094 & - & -0.389 & -0.046 & - \\
& Yellow & 0.139 & - & 0.577 & - & 0.339 \\
& Green & - & 0.436 & 0.574 & 0.059 & - \\
& Blue & - & -0.436 & -0.574 & - & -0.337 \\
& Violet & - & - & - & -0.004 & -0.002 \\
\hline$t_{j}^{o}$ & & 3.966 & 4.564 & 6.800 & 5.706 & 10.289 \\
$\bar{t}_{j}$ & & 3.667 & 4.500 & 6.400 & 7.333 & 9.667
\end{tabular}

\section{Case Study}

The waste transportation data from the particular city in the Czech Republic were used as the input for the presented model. In the preprocessing phase, the data are complemented by information about local infrastructure and the routes are divided into segments in the strategical points. Also, the outliers are removed using Grubbs or Dean-Dixon test. The data are updated and modified according to the following procedure.

\subsection{Preprocessing - Data Collection and Editing}

\section{Available data}

Vehicle movement data consisting of 325,193 different routes are available for this case study. Namely the starting and ending points of the vehicle route, driving time, distance, average and maximum speed and vehicle identification code. This information come from companies that register GPS information about routes of vehicles. It is a large database of data that needs to be adjusted and cleared from incorrect values. Some incorrect records can be identified and deleted immediately - zero time spent on the road, negative speed.

\section{Creation of probable route and separation to segments}

The available database offers information only about the start and end point of the route. The detailed path of the vehicle is unavailable. Thus, the movement of the vehicle between these positions is not known. Under the assumption about using shortest path, the route is determined using the algorithm from Leaflet JavaScript library and OpenStreetMap (OSM). The case study deals with the urban part with quite short segments, where the probable route could be estimated. The probable route is separated into segments at the crossroads. In this way, the database is enriched by a large amount of data.

The goal is to estimate the travel time on each segment. However, the transportation time depends on the type of road. All segments also store the information about road type, such as motorway, trunk, primary, 
secondary, service, etc. This classification is the basic way for minimal $t_{j}^{\text {min }}$ and maximal $t_{j}^{\text {max }}$ time setting, which are given by speed limits and traffic experience. Based on the OSM data, for each segment is assigned one type of road.

\section{Separation of unusable data}

The correspondence of the route and the real path is not ensured. The database preserves only data, where the rate of the real distance and distance from map lies within 0.9 and 1.1. In other cases, the route was probably realized along the different unknown path. In this way, the database is reduced to 84,610 routes, which means $26.02 \%$ of the original database. This significant reduction of the routes is given by problem with signal GPS or choosing of different than the shortest route (accident, traffic fees, etc.). The higher amount of applicable data would ensure the information about transit points. These routes are separated to 172,322 segments.

\section{Cluster analysis and identification of outliers}

Data could be distorted because the outliers can be found in the database. The procedure of outlier identification begins with the cluster analysis. The similar routes, in the meaning of road type (motorway, trunk, primary, etc.), are clustered in the classes. Within the individual classes, the outliers are detected using Grubbs test or Dean-Dixon test, depending on the normality of the data.

\subsection{Initialization}

According to the size of the task, the calculation is very time-consuming. It could be improved by quality initial estimations of variables included in the mathematical model Sect. 3. The starting values are given by results of the linearised model which is significantly easier to solve. The objective function (13) is derived from (9) by removing squares and weights $v_{j}$, and separating delta variables into positive and negative parts. The rest of the constraints remain the same as (2) - (11).

$$
\min \sum_{i \in I} \sum_{j \in J}\left(w_{j}\left(\delta_{i, j}^{+}+\delta_{i, j}^{-}\right)+p_{i, j}^{\max ,-}+p_{i, j}^{\min ,-}\right) .
$$

\subsection{Pseudocode}

The whole procedure of the presented approach is summarised in pseudocode 1. It starts with the initialization of starting values for the next calculation by solving the linearised form of the objective function. In step two, the non-linearised model is solved with initial values to provide more precise data for $\bar{t}_{j}$ and subsequently $v_{j}$ calculation. The final solve which utilises both weights in the non-linear objective is performed iteratively with stopping criterion $\epsilon$.

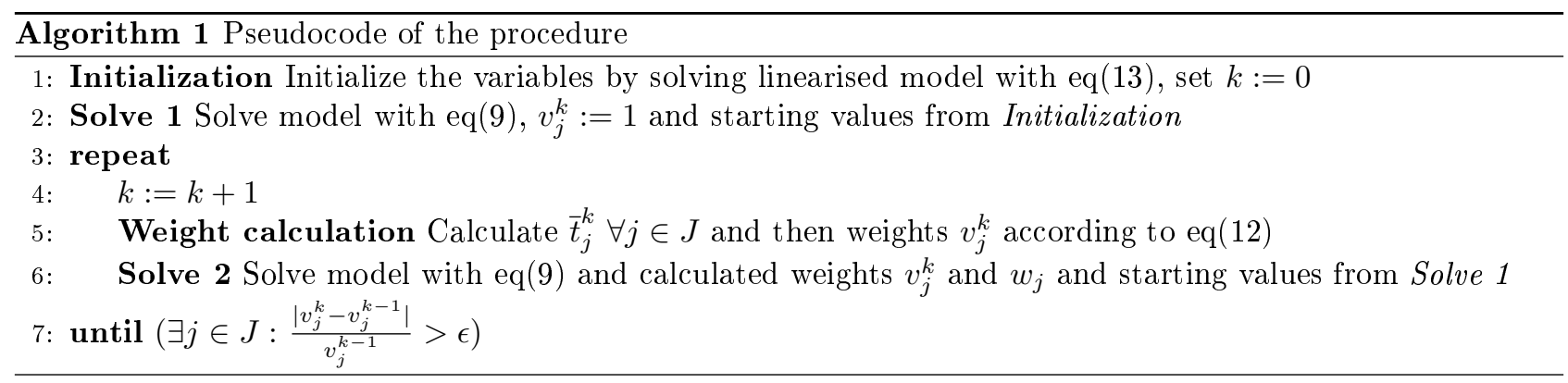

\subsection{Model Application and Results}

The mathematical model and the algorithm from previous sections are used for GPS data reconciliation from a particular area. This case study includes two applications for different territories. The parameters are summarized in the Table $5^{1}$. The mathematical model is implemented in the system GAMS (General Algebraic Modeling System) using the nonlinear CONOPT solver.

The computational time of this application forms a research gap for future improvements. As the last two rows in the Table 5 show, with the increasing number of non-zero segments, the time requirement grows significantly. The computation of larger scale tasks has to be divided into smaller tasks, for example in the form of decomposition [17]. The analysed territories are shown in the maps in the Fig. 2 (bounded by a black

\footnotetext{
${ }^{1}$ Parameters of the computer: Intel(R)Xeon(R) CPU E5-2698 v4 @ 2.20GHz 2.20 GHz, 128 GB
} 
Table 5: The summarization of computation parameters

Number of routes Territory 1

Number of segments

2,954

Territory 2

Number of non-zero elements in the matrix $t_{i, j}$

9,110

2,847

Time requirement of computation

$2 \min 35 \mathrm{~s} \quad 69 \mathrm{~h} 26 \mathrm{~min} 40 \mathrm{~s}$

color). It can be observed that both cases include urban transport network with several types of roads, as the different colours in the maps illustrate.

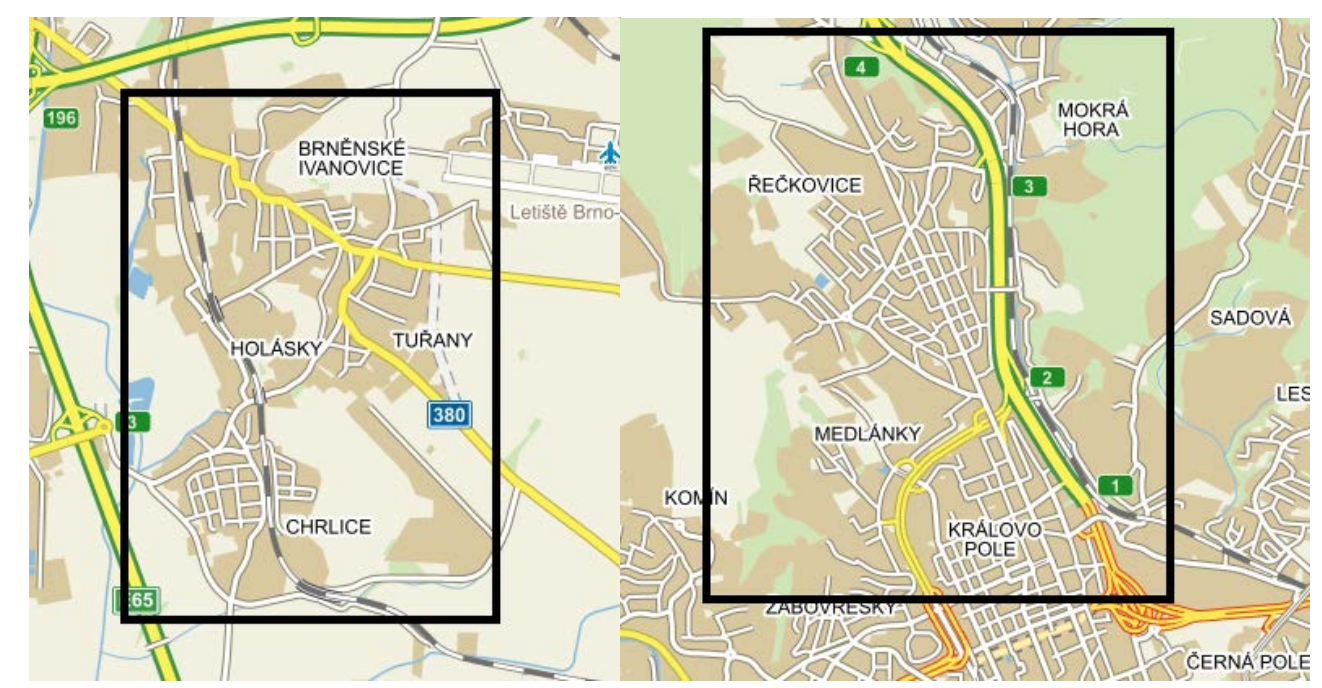

(a) Territory 1

(b) Territory 2

Figure 2: Maps of the analysed territories

The histogram in the following Fig. 3 informs about estimated speed on specific segments for Territory 2. The speed results correspond with the expectation since the most represented values lie between 0 and $50 \mathrm{~km} / \mathrm{h}$. Due to the fact that the analysed area also includes motorways and their exits, some values reach up to 130 $\mathrm{km} / \mathrm{h}$ (the class ends in 140).

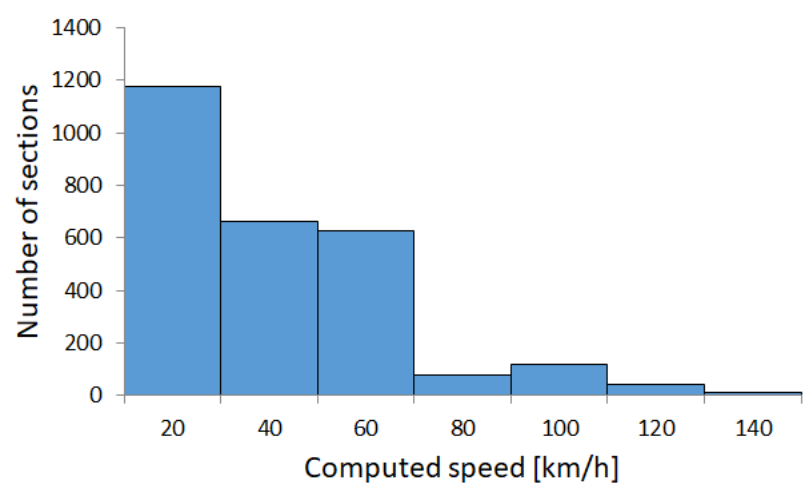

Figure 3: The histogram for estimated speed on specific segments for the Territory 2

\section{Conclusion}

The presented approach suggests the methodology for data reconciliation of large database collecting GPS data about vehicle routes. The aim is to gain the estimations of time spent on the particular segments which provide more detailed information about vehicle movement. The mathematical model is based on the weighted least squares data reconciliation. First, it is tested on simplified example, where it was managed to get very close to the expected values. The real data application carries the more complex task and requires the preparation of data set in the sense of the completion of probable overall route, maximal speed setting, outliers detection. This 
part is marked as preprocessing followed by initialization for the mathematical model. The initial estimations are given by results of the linearised model which can significantly reduce the required time. Initial values are used in the non-linear model to provide data for the calculation of weights. The calculation is then performed iteratively to update weights and thus the segment transit time with all the requisites and given stopping criterion. The procedure is demonstrated on two particular territories of the urban transport network in the Czech Republic. The possible extension for the network of the entire Czech Republic is insoluble in the acceptable computation time. In this phase of the research, the only possible approach is to separate the state data into smaller areas. The overall state computation would require the ensuring solvability, for example in the form of the advanced heuristic methodology, as previously some models have been modified [18]. The next possibility is using artificial intelligence, for example, the computational power of neural networks was discused in [19]. The extension of the processed area could be a subject of further development.

Acknowledgement: The authors gratefully acknowledge financial support provided by ERDF within the research project No. CZ.02.1.01/0.0/0.0/16_026/0008413 "Strategic Partnership for Environmental Technologies and Energy Production".

\section{References}

[1] Bong, C.P.C, Lim, L.Y., Lee, C.T., Fan, Y.V., Klemeš, J.J.: The role of smart waste management in smart agriculture. Chemical Engineering Transactions 70, 937-942 (2018).

[2] Burian, F., Florian, T., Zalud,L.: The identification of drivers behaviour through the use of GPS and odometry. Mendel 17(1), 492-496 (2011).

[3] Mazaré, P.-E., Tossavainen, O.-P., Bayen, A.M., Work D.B.: Trade-offs between inductive loops and GPS probe vehicles for travel time estimation: A Mobile Century case study. TRB, Annual Meeting, (2012).

[4] Patire, A.D, Wright, M., Prodhomme, B., Bayen A.M.: How much GPS data do we need? Transportation Research Part C: Emerging Technologies 58, 325-342 (2015). DOI: 10.1016/j.trc.2015.02.011.

[5] Narasimhan, S., Jordache, C.: Data reconciliation and gross error detection: an intelligent use of process data. Houston: Gulf Publishing Company (2000). ISBN 0-88415-255-3.

[6] Šomplák, R., Nevrlý, V., Smejkalová, V., Pavlas, M., Kůdela, J.: Verification of Information in Large Databases by Mathematical Programming in Waste Management. Chemical Engineering Transactions 61, 985-990 (2017). DOI: 10.3303/CET1761162.

[7] Fuente, M.J. , Gutierrez, G., Gomez, E., Sarabia, D., de Prada, C.: Gross error management in data reconciliation. IFAC-PapersOnLine 48(8), 623-628 (2015). DOI: 10.1016/j.ifacol.2015.09.037.

[8] Navrátilová, B., Hrdina J.: Multilateration in volumetry: Case study on demonstrator MCV 754 quick. Mendel 22(1), 295-300 (2016).

[9] Jiang, X., Liu, P., Li, Z.: Data reconciliation and gross error detection for operational data in power plants. Energy 75, 14-23 (2014). DOI: 10.1016/j.energy.2014.03.024.

[10] Cencic, O.: Nonlinear data reconciliation in material flow analysis with software STAN. Sustainable Environment Research 26(6), 291-298 (2016). DOI: 10.1016/j.serj.2016.06.002

[11] Sun, S., Huang, D., Gong, Y.: Gross Error Detection and Data Reconciliation using Historical Data. Procedia Engineering 15, 55-59 (2011). DOI:10.1016/j.proeng.2011.08.012.

[12] Isom, J.D., Stamps, A.T., Esmaili A., Mancilla, C.: Two methods of data reconciliation for pipeline networks. Computers \& Chemical Engineering 115, 487-503 (2018). DOI:10.1016/j.compchemeng.2018.05.008.

[13] Rafiee, A., Behrouzshad, F.: Data reconciliation with application to a natural gas processing plant. Journal of Natural Gas Science and Engineering 32, 538-545 (2016). DOI: 10.1016/j.jngse.2016.03.071.

[14] Doubravský, K., Dohnal, M.: Reconciliation of decision-making heuristics based on decicion trees topologies and incomplete fuzzy probabilities sets. PLoS ONE 10(7) (2015). DOI: 10.1371/journal.pone.0131590.

[15] Hrabec, D., Viktorín, A., Šomplák, R., Pluháček, M., Popela, P.: A heuristic approach to facility location problem for waste management: A case study. Mendel 22(1), 61-66 (2016).

[16] Hrabec, D., Popela, P., Roupec, J., Jindra, P., Novotný, J.: Hybrid algorithm for wait-and-see transportation network design problem with linear pricing. Mendel 21(1), 183-188 (2015).

[17] Kůdela, J., Popela, P.: Warm-start cuts for generalized benders decomposition. Kybernetika 53(6), 1012-1025 (2017).

[18] Hanna, G., Miskarik, K., Dobrovsky, L., Osmera, P.: An efficient two-level optimization method for optimal tunning of controllers. Mendel 21(1), 71-76 (2015).

[19] Šima, J.: The computational power of neural networks and representations of numbers in non-integer bases. Mendel 23(1), 103-110 (2017). 